\title{
GENOMICS
}

\section{Technical feat gives clues to human origins}

The long-awaited genome sequence of our closest evolutionary relative has now been completed. The Neanderthal genome, which was sequenced at 1.3 -fold coverage by stitching together 4 billion nucleotides of shotgun fragments, paints a revised picture of the evolutionary relationship with our ancient cousins. It also highlights functional changes that might have contributed to the success of our lineage.

The draft sequence was derived from the bones of three female Neanderthals that were dated to over 38,000 years ago. Sequencing ancient DNA is dogged by technical complications, principally sample degradation and contamination from bacteria and (more insidiously) from modern humans. The improved handling procedures used in this study — including marking ancient DNA with a unique sequence tag and using restriction enzymes to preferentially degrade bacterial DNA - ensured that contamination was $<1 \%$.

The genome sequence was obtained using Roche 454 and Illumina sequencing platforms and assembled using the human and chimpanzee reference genomes. To pick out genetic changes that evolved along the human lineage, the sequence was compared to the chimpanzee reference genome and also to the genomes of five modern humans from disparate world regions.

The authors found only 78 nucleotide changes in proteincoding genes that have become fixed in humans since they diverged from Neanderthals. These variants occur in genes with intriguing functions, such as skin physiology. Over 250 fixed regulatory variants were also detected, including one that might affect microRNA target specificity. But which of these fixed changes were important for the making of modern humans? The authors identified 20 regions with a strong signal of positive selection on the modern human lineage, encompassing genes relevant to metabolism, skeletal morphology and cognitive function.

The low sequence coverage of the shotgun genome means that about one-third of the genome is unavailable for studying. To address this limitation, Burbano and colleagues carried out targeted sequencing on the Neanderthal sample - the first time this approach has been performed on ancient DNA. Tiled microarrays were used to capture over $1 \mathrm{Mb}$ of Neanderthal DNA

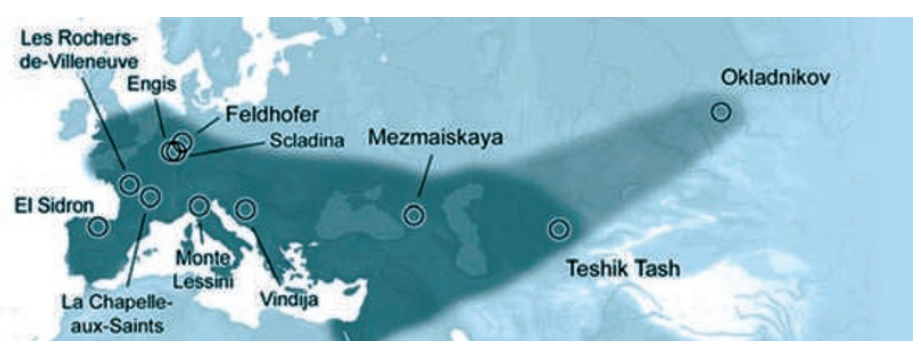

The geographical range of Neanderthals. Image is reproduced, with permission, from Krause, J. et al. Neanderthals in central Asia and Siberia. Nature 449, 902-904 (2007) @ Macmillan Publishers Ltd. All rights reserved.

containing non-synonymous substitutions that have occurred in the shared Neanderthal/human lineage since the ancestral split from chimpanzees. By comparing these 14,000 sites to those in 50 modern humans, the authors detected 88 substitutions that are fixed in modern humans — sites that will undoubtedly merit functional investigation.

SNP comparisons between Neanderthals and modern humans also shed light on the long debated issue of whether Neanderthals interbred with modern humans: it turns out that Neanderthals are more closely related to (that is, share more SNPs with) Europeans and East Asians than sub-Saharan Africans. This unexpected result was thoroughly validated, and is most consistent with gene flow having occurred from Neanderthals to modern humans after the latter left Africa but before they migrated to Eurasia. The amount of genetic mixing was probably modest, however, as only $1-4 \%$ of the genome of modern Eurasians derives from the Neanderthal.

Technological leaps have made it feasible to reliably reconstruct the genome of an ancient human. Analyses of this and additional genomes will follow up on these hypotheses and will almost certainly generate more.

Tanita Casci

ORIGINAL RESEARCH PAPERS Green, R. E. et al A draft sequence of the Neandertal genome. Science 328, 710-722 (2010)|Burbano, H. A. et al. Targeted investigation of the Neandertal genome by array-based sequence capture. Science 328, 723-725 (2010) WEBSITE

Special feature on the Neanderthal genome in Science: http://www.sciencemag.org/special/ neandertal 